Hydroécol. Appl. (2000) Tome 12 Vol. 1-2, pp. 183-206

\title{
Étude du régime thermique de la retenue Al Massira (Maroc)
}

\section{Thermal regime of Al Massira reservoir (Morocco)}

\author{
L. Alaoui ${ }^{\star}$, A. Agoumi ${ }^{\star *}$, M. Moncef*, K. Mokhliss ${ }^{\star}$ \\ (*) Laboratoire d'Hydrobiologie. Faculté des Sciences. Université Chouaib Doukkali.B.P. 20 El \\ Jadida, Maroc. \\ ${ }^{* \star}$ Ecole Hassania des Travaux Publics, $\mathrm{Km} 7$, route d'Al Jadida. Casablanca, Maroc.
}

Résumé. - Les différentes formes de pollution auxquelles les eaux de surface du Maroc ont été soumises ces dernières années, associées au climat semi-aride à aride de nos régions, ont entraîné un enrichissement important de ces eaux en éléments nutritifs avec pour conséquence une menace d'eutrophisation des retenues de barrages.

L'altération des eaux résultant de ce phénomène d'eutrophisation est particulièrement complexe. Une simple analyse et un traitement statistique des données recueillies in situ ne permet pas de concevoir une approche globale de la qualité des eaux de ces systèmes aquatiques en particulier des lacs et réservoirs : une approche par modélisation s'impose.

Ainsi, et dans une première étape de modélisation de la dynamique d'eutrophisation de ces écosystèmes, un modèle thermique a été élaboré, en vue de simuler pour la retenue Al Massira, l'évolution saisonnière de la température et de la structure hydrodynamique verticale.

C'est un modèle bicouche à bilan d'énergie, basé sur la représentation des mécanismes physiques, qui prend en compte les échanges d'énergie à l'interface air-eau ainsi que les effets des entrées et sorties d'eau. Les résultats de cette tentative de modélisation sont analysés dans ce travail. Une étude de sensibilité de ce modèle aux différents paramètres y est également présentée.

Ce modèle thermique sera couplé dans une prochaine étape avec un modèle biologique pour simuler l'évolution de la qualité des eaux dans cette retenue.

Mots clés. - retenue, modélisation thermique, bicouche

Abstract. - Different forms of pollution that surface water in Morocco has been subjected to in recent years, associated with the arid climate in the area, have resulted in significant nutrient inputs in these waters and led to eutrophication of the reservoirs.

The change in water quality as a result of this eutrophication phenomenon is particularly complex. Simple analysis and statistical processing of the data in the field do not enable designing a global approach to the water quality of these aquatic systems, particularly 
the lakes and reservoirs : modelling is necessary.

In the first phase of the modelling of the dynamics of eutrophication, a thermal model was developed to simulate seasonal evolution of the temperature and hydrodynamic vertical structure in Al Massira reservoir.

This two-layer model is an energy budget model based on representation of physical mechanisms, taking into account the surface energy exchanges and throughflow in the reservoir. The results of this modelling attempt are analysed. A study of sensitivity of this model to the various coefficients is also presented.

In the future, this thermal model will be coupled with a biological model in the second phase of this project, to simulate evolution in the quality of AI Massira water.

Key words. - reservoir, thermal modelling, two-layered

\section{INTRODUCTION}

Dans un lac, le sous-système physique conditionne l'évolution du soussystème écologique et en détermine largement le fonctionnement. La température joue un rôle primordial dans le mouvement des masses d'eau. Elle représente ainsi une variable essentielle dans la représentation physique du système.

La température est en plus un facteur déterminant pour la dynamique écologique de ces systèmes aquatiques puisqu'elle influence fortement les cinétiques chimiques et biologiques.

Ainsi toute étude de l'évolution écologique et de l'eutrophisation des lacs et réservoirs nécessite au préalable une étude thermique.

Dés 1989, le barrage Al Massira, a fait l'objet d'une étude pluridisciplinaire lancée par une équipe d'universitaires (Moncef, 1993; Malki, 1994; Fahde, 1994 ; Dafir, 1997 et Mokhliss et Alaoui à paraître), dans le but d'appréhender la cinétique d'eutrophisation de cette retenue et le déve- loppement planctonique dans son contexte physique et géochimique. Une base de données relatives à la qualité des eaux a été recueillie lors des nombreuses campagnes de mesures sur le terrain.

Le travail de recherche dont la première partie est présentée ici a concerné l'essai de modélisation de l'eutrophisation des eaux dans ce barrage, utilisant les données recueillies in situ par les auteurs précités.

La modélisation de la température a constitué la première étape de la modélisation entreprise et a été menée de façon totalement indépendante de la modélisation du sous-système écologique.

Après une analyse des données de terrain, le modèle est construit et ajusté sur l'échantillon de mesures disponibles. La base de données utilisée concerne trois cycles annuels en 1990,1991 et 1997 .

Une étude de sensibilité a été faite. Elle a permis d'estimer l'importance relative des différents paramètres intervenant dans la modélisation de la dynamique de cet écosystème. 


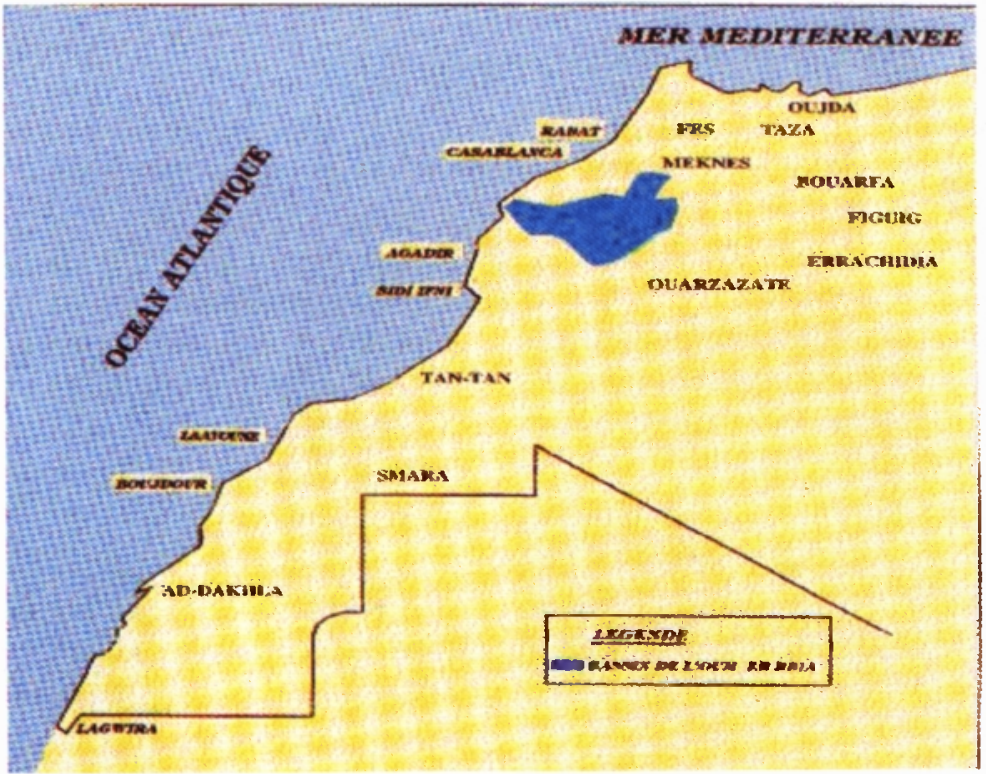

Carte 1 - Situation du bassin de l'Oum Er Rbia sur la carte du Maroc. Carte 1 - Location of Oum Er Rbia watershed in Morocco.

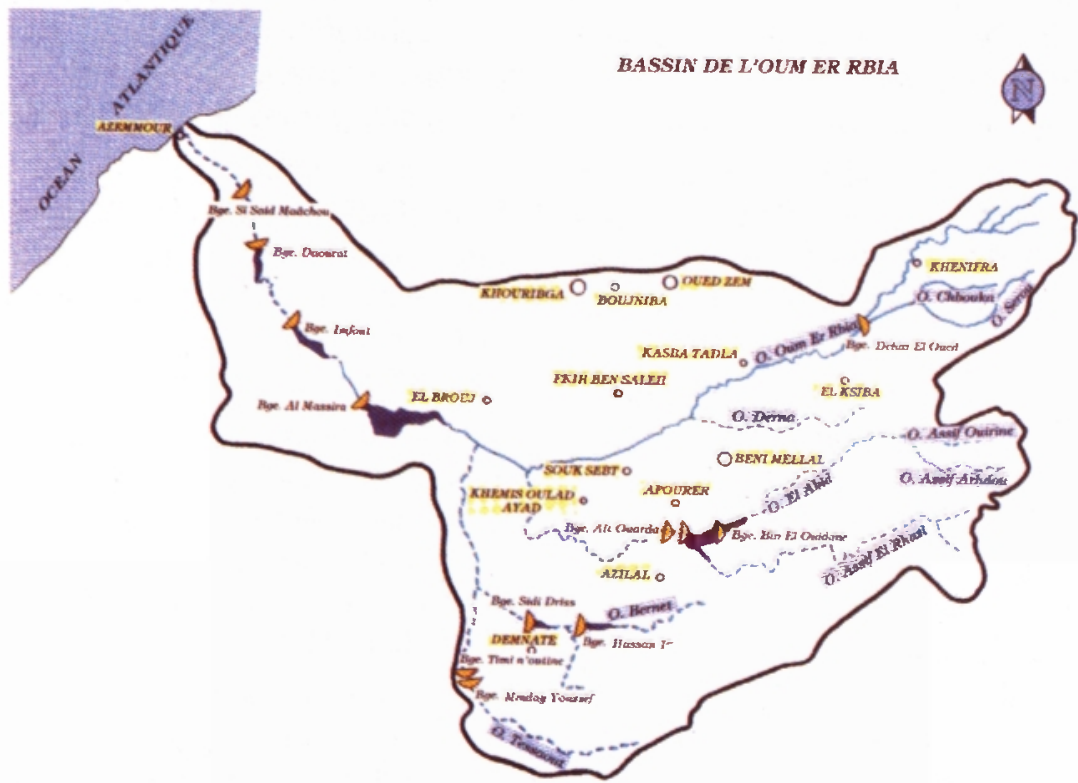

Carte 2 - Situation de la retenue Al Massira sur le bassin versant de l'Oum Er Rbia. Carte 2 - Location of Al Massira reservoir in Oum Er Rbia watershed. 


\section{CARACTÉRISTIQUES DE LA RETENUE}

Située à 120 Kilomètres environ au sud-est de Casablanca (cartes 1 \& 2), la retenue Al Massira est l'une des plus grandes retenues marocaines, à usages multiples : eau potable, agriculture, industrie et énergie. Les caractéristiques de la retenue sont représentées sur le tableau I.

Construite sur l'oued Oum Er Rbia, la retenue du barrage Al Massira est située dans l'étage bioclimatique aride à hiver tempéré (Emberger, 1955). Le climat au niveau de la retenue présente un caractère continental, marqué par une grande amplitude thermique et une insolation forte, (Malki, 1994). Deux facteurs agissant en sens contraire, précipitations et température conditionnent le climat semi-aride de cette région.

Les variations saisonnières du volume d'eau de la retenue Al Massira sont parfois importantes; elles sont dues aux épisodes de sécheresse fréquents et de longues durées et résultent :

- des apports liquides par l'oued Oum Er Rbia,

- et des restitutions (notamment soutirages de l'Office National d'Electricité) et de l'évaporation (Malki, 1994).

Les principales données ayant servi à l'analyse de l'évolution de la qualité des eaux de la retenue Al Massira ont pour origine :

- La Direction de la Recherche et de la Planification de l'Eau (DRPE).

- Le programme pluridisciplinaire lancé par une équipe d'universitaires : Moncef, Malki et Fahde (19891994).

Ces différentes campagnes de mesures ont permis de cerner les principales caractéristiques du réservoir :

\subsection{Température}

La retenue Al Massira appartient à la catégorie des lacs monomictiques

Tableau I. - Caractéristiques principales de la retenue AI Massira.

Table I. - Principal characteristics of the Al Massira reservoir.

\begin{tabular}{|l|c|}
\hline Paramètres & Valeurs \\
\hline Année de mise en eau & 1979 \\
Surface du bassin versant $\left(\mathrm{km}^{2}\right)$ & 28500 \\
Profondeur maximale $(\mathrm{m})$ & 40 \\
Profondeur moyenne à la cote normale $(\mathrm{m})$ & 20 \\
Longueur $(\mathrm{km})$ & 30 \\
Largeur maximale $(\mathrm{km})$ & 10 \\
Cote normale (NGM) & 285 \\
Cote maximale (NGM) & 287,5 \\
Cote de la prise d'eau (NGM) $\left({ }^{*}\right)$ & 240 \\
Volume au niveau normal $\left(10^{6} \mathrm{~m}^{3}\right)$ & 2800 \\
Temps de séjours (mois) & $10-42$ \\
\hline
\end{tabular}

(*) ie $+10 \mathrm{~m}$ au-dessus du fond de la retenue (230 NGM). 
chauds. Elle connaît l'été une légère stratification thermique de l'ordre de $2{ }^{\circ} \mathrm{C}$. Les écarts entre la température de surface et celle du fond sont très réduits (maximum de $5{ }^{\circ} \mathrm{C}$ en juillet 1991). Cette situation est à associer aux turbulences hydrodynamiques engendrées par l'ouverture des vannes de sorties, auxquelles s'ajoute l'action des vents souvent violents qui soufflent en toute saison sur la retenue, (Malki, 1994).

\subsection{Paramètres chimiques}

L'utilisation des eaux de l'oued Oum Er Rbia et de ses différentes retenues, a permis de développer d'importantes activités économiques dans le secteur agricole, agro-industriel et urbain (environ 325000 habitants).

Outre les apports d'origine domestique ( 9000 t/an de MO (matières oxydables), $\sim 6500$ t/an de MES(matières en suspension) et $\sim 1100$ t/an de matières azotées (ONEP, 1987), l'ensemble de ces activités s'est accompagné d'un accroissement rapide des rejets polluants de nature industrielle (tanneries, textiles) et agricole (sucreries, huileries) de l'ordre de $8000 \mathrm{t} /$ an de matières oxydables et 7500 t/an de matières en suspension (DH, 1990).

L'analyse des résultats sur la période 1980-1985 montre que le lac-réservoir Al Massira présente des symptômes révélateurs de la dégradation de la qualité de l'eau tels que le déficit en oxygène dans l'hypolimnion (Abouzaid et al, 1987).

En 1990, la retenue a atteint un certain équilibre au niveau de son comportement écologique avec :

- des teneurs en oxygène dissous ne s'abaissant que très rarement au-dessous de $5 \mathrm{mg} / \mathrm{l}$ quelle que soit la station considérée ;

- des valeurs de $\mathrm{pH}$ le plus souvent maximales dans les dix premiers mètres, ce qui traduit l'influence de l'activité photosynthétique sur les valeurs de ce paramètre;

- des teneurs en azote et phosphore extrêmement variables en liaison avec les fluctuations de l'intensité des apports par les rejets industriels en amont du barrage, et du métabolisme algal;

- des concentrations en phosphore soluble relativement faibles pour un lac dont l'une des rives est située sur des terrains à phosphate.

\subsection{Analyse du phytoplancton et du zooplancton}

Les prélèvements effectués entre 1989 et 1992, ont permis de décrire la dynamique des populations algales et zooplanctoniques :

- Les travaux de Malki (1994) avaient montré que le peuplement phytoplanctonique, qualitativement et quantitativement est relativement homogène sur les vingt premiers mètres de la colonne d'eau. Il est essentiellement composé de Dinophycées (Peridinium cinctum, Ceratium hirun- 
dinella...), de Chloropycées (Oocystis crassa, Scenedesmus ecoenis...) et de Diatomées (Cyclotella sp.), les densités cellulaires étant très variables d'une espèce à l'autre.

En été, la retenue Al Massira présente une importante biomasse phytoplanctonique. Celle ci est essentiellement associée à la présence de populations de Dynophycées qui bien que présentant des effectifs réduits, constituent un biovolume considérable en raison de leur volume cellulaire.

- La communauté zooplanctonique, dont la densité moyenne, déterminée par dénombrement sur eau brute est de 46 individus par litre, est essentiellement composée de Copépodes $(50,4 \%$ de l'effectif total) et de Cladocères $(47,6 \%)$, les Rotifères ne constituant qu'une fraction minime (2\%) de l'ensemble. Du point de vue qualitatif :

- les Copépodes sont représentés par le Calanoïde Neolovenula alluaudi $(81,5 \%)$ et les Cyclopoïdes Acanthocyclops robustus (16,5\%) et à un degré moindre Tropocyclops prasinus $(2 \%)$. La biomasse maximale des Copépodes est d'en moyenne de $1,2 \mathrm{mg}$ de poids sec $/ 1$. Les valeurs les plus élevées sont observées chaque année entre avril et mi-Juillet puis de septembre à novembre (Moncef, 1993).

- les Cladocères sont représentés par Diaphanosoma brachyurum (espèce dominante), Daphnia Iongispina et Ceriodaphnia reticulata
(Moncef et al, 1994). Parallèlement au développement des copépodes, les cladocères atteignent les effectifs les plus élevés en été et de septembre à la mioctobre. Le peuplement de Rotifères de la retenue Al Massira, bien que relativement diversifié, présente une biomasse faible au sein du peuplement zooplanctonique. (Fahde, 1994).

Le zooplancton présente donc deux périodes de développement, une première période allant du printemps jusqu'au début de l'été et une autre en automne.

\section{MODÈLE THERMIQUE ADOPTÉ}

\subsection{Structure du modèle}

Le cycle thermique saisonnier d'un lac dépend essentiellement des apports d'énergies thermique et mécanique au plan d'eau et leur transport dans la colonne d'eau. La mise en place de la stratification est un phénomène majeur qui a plusieurs conséquences sur le fonctionnement global et, en particulier écologique du lac. La température de l'eau en tant que facteur physique, reste l'un des éléments les plus importants de l'écosystème puisqu'elle conditionne la cinétique de la plupart des réactions chimiques et des processus biologiques.

Ainsi, l'évolution écologique de l'écosystème Al Massira est fortement liée au régime thermique et hy- 
drodynamique des eaux. D'où l'importance d'élaborer en première partie un modèle de simulation de l'évolution thermique des eaux de ce réservoir.

Pour la retenue Al Massira, le modèle proposé est inspiré des modèles mathématiques développés pour le lac de Pareloup par Salençon (1986), Thébault et Salençon (1993) et pour la Manche par Agoumi (1982, 1985).

Le modèle thermique élaboré dans cette étude est un modèle bicouche à bilan d'énergie. Les modèles à bilan d'énergie (type intégral) sont souvent adaptés à la modélisation de la zone bien mélangée de surface. La température et la profondeur sont calculées de telle sorte que l'énergie cinétique turbulente induite par le vent et la variation d'énergie potentielle induite par les forces gravitationnelles de la zone bien mélangée soient en équilibre.

Dans ce modèle les deux couches sont d'épaisseurs variables. La couche de surface ou épilimnion, est turbulente et est séparée de la couche de fond, non turbulente ou hy- polimnion, par une thermocline. Chaque couche est caractérisée par sa température, son volume, et sa profondeur. Les échanges hydrodynamiques entre les deux masses d'eaux sont représentés par une vitesse d'entraînement de la couche de fond vers la couche de surface (fig. 1).

L'évolution spatio-temporelle de la température des lacs- réservoirs est en général conditionnée par quatre phénomènes (fig. 2) :

- Les échanges énergétiques à l'interface eau-atmosphère: il s'agit d'échanges convectifs et radiatifs pouvant être estimés à partir des données météorologiques locales.

- Pénétration sélective du rayonnement solaire avec un apport énergétique important associé aux eaux de surface par rapport aux eaux profondes.

- L'effet mécanique engendré par le cisaillement du vent à la surface libre qui engendre la formation d'une couche mélangée chaude et homogène séparée des eaux pro-
$T_{11} T_{2}$ : Températures de surface et de fond $H_{1}, H_{2}$ : Profondeurs de surface et de fond $V_{1}, V_{2}$ : Volumes de surface et de fond

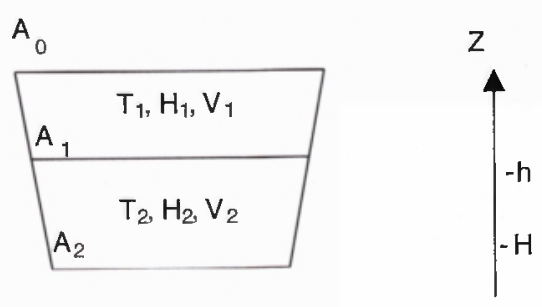

Fig. 1. - Schéma du modèle.

Fig. 1. - Model diagram 


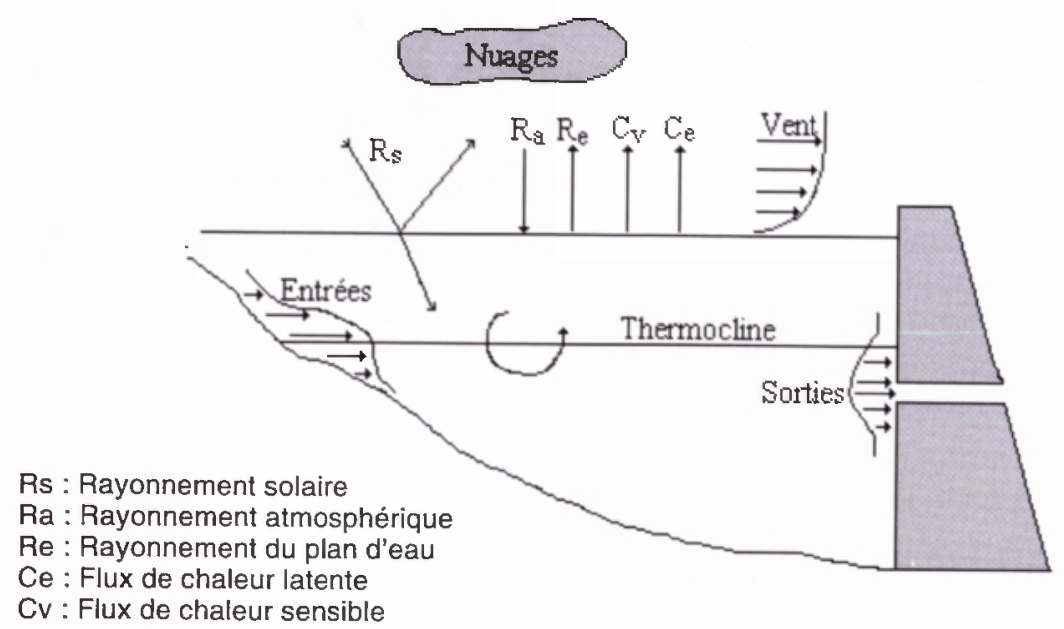

Fig 2. - Bilan d'énergie. d'une retenue et dynamique interne Fig 2. - Energy budget and internal dynamics of a reservoir

fondes froides par une barrière de densité : la thermocline.

- Les échanges hydrodynamiques résultant des processus d'entrées et de soutirages des eaux.

\subsection{Equations du modèle thermique}

Les variables qui définissent dans le temps et dans l'espace l'état dynamique, thermodynamique et biochimique du milieu étudié sont (Delclaux, 1980; Agoumi, 1982; Cazelle, 1987) :

$\rho$ : masse volumique.

$\mathrm{P}$ : pression.

$T$ : température.

$\mathrm{U}(\mathrm{U}, \mathrm{V}, \mathrm{W})$ : vecteur vitesse.
La modélisation adoptée au niveau de la retenue Al Massira repose sur les hypothèses suivantes :

- La température est homogène dans chacune des deux couches.

- L'homogénéité horizontale: les gradients thermiques horizontaux dus à l'advection sont négligés par rapport à la stratification verticale, l'advection thermique étant représentée explicitement avec un sous-modèle du premier ordre. Ceci se traduit dans l'équation d'enthalpie simplifiée sans advection thermique (Lesaos, 1979) par :

$$
\rho C_{p} \frac{\partial \bar{T}}{\partial t}=\frac{\partial}{\partial z}\left(\rho_{0} C_{p} \overline{T^{\prime} W^{\prime}}\right)+R_{z}
$$

où

$\rho_{0}$ : densité du milieu; 
$\mathrm{C}_{\mathrm{p}}$ : chaleur spécifique de l'eau;

$T$ et $W$ représentent la température moyenne et la vitesse moyenne, $T^{\prime}$ ' et $W^{\prime}$ leurs fluctuations respectives;

$\mathrm{R}_{\mathrm{z}}$ : rayonnement solaire pénétrant à la profondeur $\mathrm{z}$ au voisinage de la surface.

$R_{z}=R_{s} e^{\text {(eta"z) }}\left(R_{z}\right.$ en W.m $\left.m^{-2}\right)$

$z$ : profondeur (en $\mathrm{m}) \mathrm{z}<0$.

et a : coefficient d'extinction de la lumière dans l'eau $\left(\mathrm{m}^{-1}\right)$.

- Structure thermique verticale: nous supposons que l'énergie cinétique turbulente $(\mathrm{ECT})$ est quasi-stationnaire :

$$
\frac{d \overline{e^{2}}}{d t}=0
$$

Nous obtenons après simplification aux moments d'ordre 2, l'équation suivante :

$$
\begin{aligned}
& -\rho_{0}\left(\overline{U^{\prime} W^{\prime}} \frac{\partial \bar{U}}{\partial z}+\overline{V^{\prime} W^{\prime}} \frac{\partial \bar{V}}{\partial z}\right)-g \overline{\rho^{\prime} W^{\prime}} \\
& -\frac{\partial}{\partial z}\left(\overline{P^{\prime} W^{\prime}}+\overline{e^{2} W^{\prime}}\right)-\frac{\mu}{2}\left(\frac{\partial U_{i}^{\prime}}{\partial X_{i}}+\frac{\partial U_{j}^{\prime}}{\partial X_{i}}\right)=0
\end{aligned}
$$

g : gravité

$\mu$ : coefficient de viscosité moléculaire, de la quantité de mouvement.

Les courants de fond étant très faibles en retenues, nous les négligeons et de ce fait nous considérons que la dynamique de la masse d'eau est régie par la seule vitesse d'entraînement de la couche de fond vers celle de surface. A l'aide de ces hypothèses simplificatrices, l'évolution thermique des masses d'eau est obtenue en intégrant les équations de masse et d'enthalpie sur les deux couches et l'équation de l'ECT sur la couche de surface.

\subsubsection{Equation d'état}

Pour suivre l'évolution de la masse volumique en fonction de la température, nous avons utilisé la relation linéaire classique car la température de l'eau est toujours supérieure à $4{ }^{\circ} \mathrm{C}$ :

$$
\rho=\rho_{0}(1-\alpha T)
$$

$\rho_{0}=10^{3} \mathrm{~kg} \cdot \mathrm{m}^{-2}$;

$\alpha$ : coefficient d'expansion thermique de l'eau.

\subsubsection{Equation de continuité (conservation de la masse)}

Pour chaque couche de la structure verticale considérée, l'équation de la conservation de la masse est obtenue en faisant un bilan massique avec un modèle du premier ordre pour l'advection. La variation de volume est liée aux débits apportés et soutirés :

$$
\frac{\partial V_{j}}{\partial t}=Q_{\text {appj }}-Q_{\text {souj }}
$$

$V_{j}$ : volume de la couche $j$

$Q_{\text {app] }}$ : débit apporté à la couche $j$

$Q_{\text {soutj }}$ : débit soutiré à la couche j

En ce qui concerne les entrées, deux cas sont envisagés en fonction des températures de l'affluent $\left(T_{\text {app }}\right)$ et de la couche de surface (T1); si $\mathrm{T}_{\mathrm{app}}>$ $\mathrm{T}_{1}$, l'entrée d'eau aura lieu dans la couche de surface $\left(Q_{\text {app1 }}=Q_{\text {app }} ; Q_{\text {app2 }}=\right.$ 0 ) et si $T_{\text {app }}<T_{1}$, elle aura lieu dans la couche de fond $\left(Q_{\mathrm{app} 2}=Q_{\mathrm{app}} ; Q_{\mathrm{app} 1}=0\right)$. 
Le soutirage est considéré, au travers d'hypothèses simplificatrices, comme réparti entre les deux couches, mais avec un coefficient de pondération pris comme facteur d'ajustement du modèle. Ce coefficient traduit le fait que l'on soutire davantage d'eau de la couche (épilimnion ou hypolimnion) située au niveau de la prise d'eau, en fonction de l'évolution annuelle de la position de la thermocline.

\subsubsection{Entraînement à l'interface entre les deux couches}

La turbulence introduite dans la couche de surface par le vent et le refroidissement provoque l'accroissement de l'épaisseur de la couche de surface par entraînement d'une partie de la couche de fond.

La vitesse d'entraînement We est obtenue après intégration de l'équation de l'ECT sur la couche de surface par :

$W_{e}=\frac{1}{\left(T_{1}-T_{2}\right)}\left[\frac{2 m_{v} U_{v}^{3}}{g o h}-(1-n) \frac{P+|P|}{2 \rho_{0} C_{p}}\right]$ $+\frac{P}{\rho_{0} C_{p}}+\frac{R(0)+R(-h)}{\rho_{0} C_{p}}-\frac{2}{\rho_{0} C_{p} h} \int_{h}^{0} R(z) d z$

avec :

$n$ et $m_{v}$ : coefficients de paramétrisation du mode de production de l'ECT.

$h$ : profondeur de la couche de surface.

On définit le terme $P$ : pertes en surface $P=R_{E}+C_{v}+C_{E}-R_{A}$

$R_{E}$ : rayonnement à la surface de l'eau. $R_{A}$ : rayonnement atmosphérique.
$\mathrm{C}_{\mathrm{v}}$ : flux de chaleur sensible.

$\mathrm{C}_{\mathrm{E}}$ : flux de chaleur latente.

$T_{1}$ et $T_{2}$ sont respectivement la température de la couche de surface et de la couche de fond.

$\mathrm{R}(\mathrm{z})$ : flux radiatif arrivant à la profondeur $z(R(z)<0$ par convention de signe).

La variation de volume de la couche de surface $V_{1}$ est calculée par :

$$
\frac{\partial V_{1}}{\partial t}=W_{e} \cdot A
$$

$A$ : section de l'interface surface-fond $W_{e}$ : vitesse d'entraînement de la couche de fond vers la couche de surface.

\subsubsection{Equation d'enthalpie}

L'équation d'enthalpie intégrée donne :

* sur la couche 1 de surface :

$\frac{\partial T_{1}}{\partial t}=\left(\frac{W_{e-1}}{h}\right)\left(T_{2}-T_{1}\right)+\frac{R(-h)-R(0)-P}{\rho_{0} C_{p} h}$

* sur la couche 2 de fond:

$$
\frac{\partial \mathrm{T}_{2}}{\partial \mathrm{t}}=\frac{\mathrm{R}(-\mathrm{H})-\mathrm{R}(-\mathrm{h})}{\rho_{0} \mathrm{C}_{\mathrm{p}}(\mathrm{H}-\mathrm{h})}
$$

L'intégration de l'équation d'enthalpie sur les deux couches a été faite dans notre cas, avec l'hypothèse simplificatrice que la retenue est à sections constantes. Ceci n'est qu'une approximation pour le cas de la retenue Al Massira, dont les sections varient avec la profondeur.

$Q_{\text {app } 1}$ (resp. $Q_{\text {app } 2}$ ) représentent la part du débit qui entre dans la couche de surface (resp. de fond). 


$$
\begin{aligned}
& * \frac{\partial V_{i}}{\partial t}=Q_{a p p 2}-Q_{\text {sou2 }} \\
& * \frac{d V_{1}}{d t}=Q_{a p p 1}-Q_{\text {sou1 }}+A_{1} \cdot W_{e} \\
& * \frac{\partial V_{1} T_{1}}{\partial t}=Q_{a p p 1} T_{1}-Q_{\text {sou1 }} T_{1}+W_{e} T_{2} A_{1}+\frac{\left(R(-h) \cdot A_{1}\right)-\left(R(0) \cdot A_{0}\right)-\left(P \cdot A_{0}\right)}{\rho_{0} C_{p}} \\
& * \frac{\partial V_{2} T_{2}}{\partial t}=Q_{a p p 2} T_{2}-Q_{\text {sou2 }} T_{2}-W_{e} T_{2} A_{1}+\frac{\left(R(-H) \cdot A_{2}\right)-\left(R(-h) \cdot A_{1}\right)}{\rho_{0} C_{p}} \\
& * W_{e}=\frac{1}{\left(T_{1}-T_{2}\right)}\left[\frac{2 m_{v} U_{v}^{* 3}}{g \alpha h}-(1-n) \frac{P+|P|}{2 \rho_{0} C_{p}}+\frac{P}{\rho_{0} C_{p}}+\frac{R(0)+R(-h)}{\rho_{0} C_{p}}-\frac{2}{\rho_{0} C_{p} h} \int_{h}^{0} R(z) d z\right]
\end{aligned}
$$

$Q_{\text {sout }}$ (resp. $Q_{\text {sou2 } 2}$ ) représentent la part du débit qui en sort.

$\mathrm{V}, \mathrm{V}_{1}$ et $\mathrm{V}_{2}$ sont respectivement le volume total de la retenue, le volume de la couche de surface et celui de la couche du fond.

La résolution numérique est effectuée en utilisant le schéma numérique en différences finies explicite d'Euler.

\section{APPLICATION DU MODÈLE BICOUCHE À LA RETENUE AL MASSIRA}

Le modèle thermique bicouche développé a été appliqué à la retenue $\mathrm{Al}$ Massira pour les années 1990, 1991 et 1997.

L'échelle de temps des phénomènes reproduits est d'une journée. Le système d'équations différentielles constituant le modèle est résolu avec un pas d'intégration de trois heures qui correspond au temps de saisie des données météorologiques $(\Delta t=3$ heures).

\subsection{Les données d'entrée}

\subsubsection{Les données topographiques}

- Courbes cote/volume, cote/surface.

La morphologie de la retenue est introduite dans le modèle par le biais des courbes cote/volume et cote/surface d'Al Massira (DGH, 1990) (fig. 3).

- Niveau de soutirage

La prise d'eau se trouve à 10 mètres du fond de la retenue.

\subsubsection{Les données météorologiques}

Le modèle utilise des données trihoraires des paramètres météorologiques suivants : la vitesse du vent 
à 10 mètres de la surface $\left(m \cdot s^{-1}\right)$, la température de l'air en ${ }^{\circ} \mathrm{C}$, l'humidité relative (\%), la pression atmosphérique (mbar) et la nébulosité (octas).

Les données météorologiques utilisées sont celles de la station de Béni Mellal. Le choix de cette station a été fait en se basant sur sa proximité par rapport à la retenue $\mathrm{Al}$
Massira et du fait que cette station est située dans la même zone climatique.

\subsubsection{Les données de débits}

Les données de débits d'entrée d'eau sont celles de l'oued Oum Er Rbia à l'amont de la retenue Al Massi-

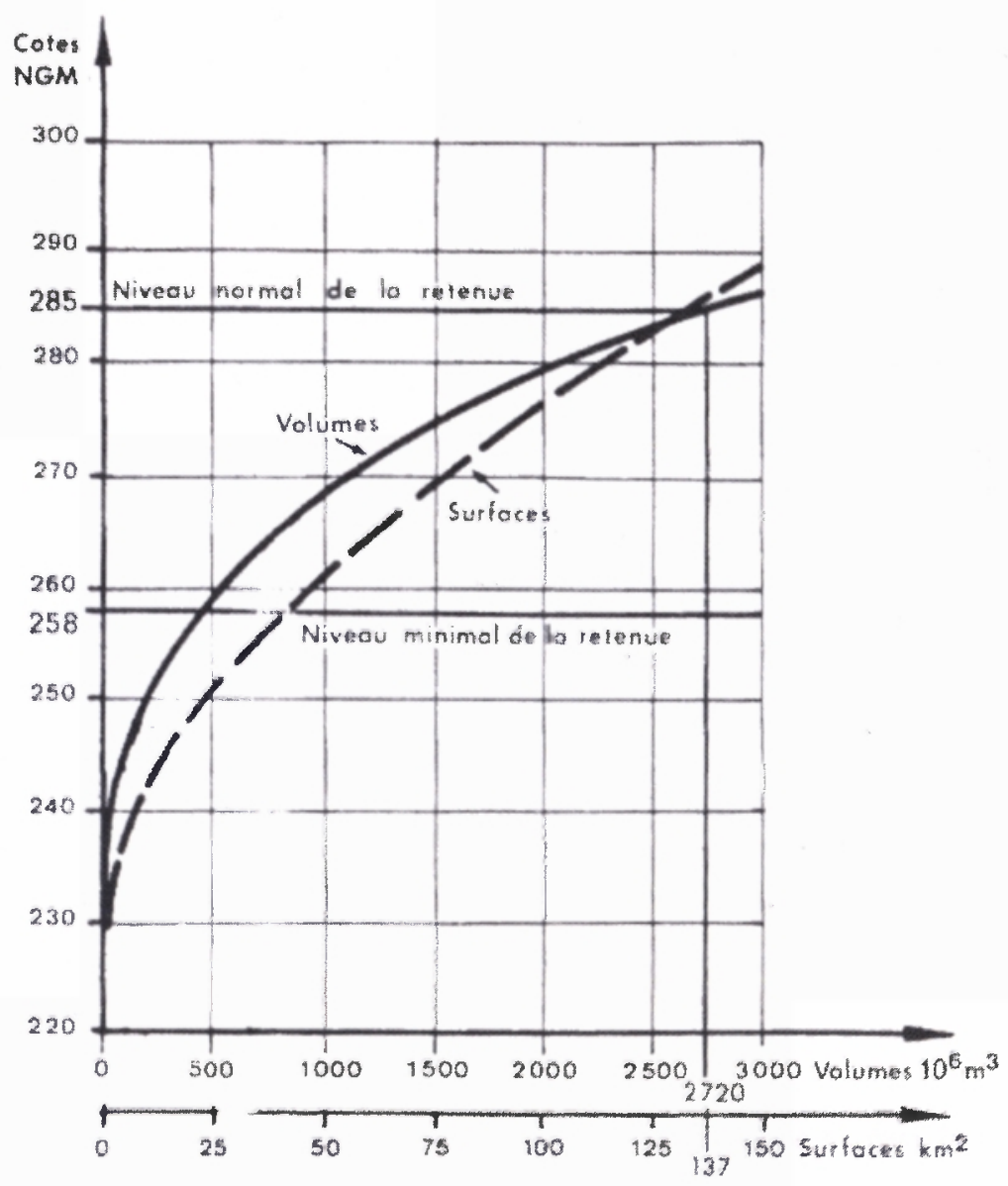

Fig. 3. - Courbes cote/volume et cote/surface de la retenue Al Massira. Fig. 3. - Level/volume and level/surface curves for Al Massira reservoir. 
ra. Elles correspondent aux mesures effectuées par la DRPE durant les années 1990, 1991 et 1997.

Les données des débits de sorties sont celles de la prise d'eau relevées par l'Office National d'Electricité pour les mêmes années. Pour l'année 1990, les débits journaliers des apports et des soutirages sont représentés sur la figure 4 .

\subsubsection{La température des apports}

Il est particulièrement important de bien reproduire la température des apports car c'est elle qui détermine par effet de densité, la profondeur à laquelle chaque entrée d'eau s'insère dans la retenue.

Dans notre étude les températures de l'affluent sont calculées avec un modèle thermique de rivière (modèle zérodimensionnel; Bensaid, 1994) appliqué à l'amont de la retenue $\mathrm{Al}$ Massira. Ce dernier reproduit de manière satisfaisante les niveaux de températures de l'eau en amont de la retenue pour le printemps et l'été, mais les sous-estime en automne et en hiver (fig. 5).

\subsection{Les données de calage}

Les profils thermiques qui ont été utilisés pour le calage du modèle thermique correspondent à des mesures effectuées au même endroit avec un pas d'échantillonnage le plus souvent bimensuel. Les prélèvements ont été réalisés aux profondeurs suivantes: surface, $2 \mathrm{~m}, 5 \mathrm{~m}$, $10 \mathrm{~m}, 15 \mathrm{~m}, 20 \mathrm{~m}, 25 \mathrm{~m}, 30 \mathrm{~m}$ et prés du fond (à environ $35 \mathrm{~m}$ ). La température de l'eau a été mesurée dans la bouteille de prélèvement, immédiatement après la collecte, à l'aide d'un thermomètre à mercure donnant une précision de $\pm 0,1^{\circ} \mathrm{C}$.
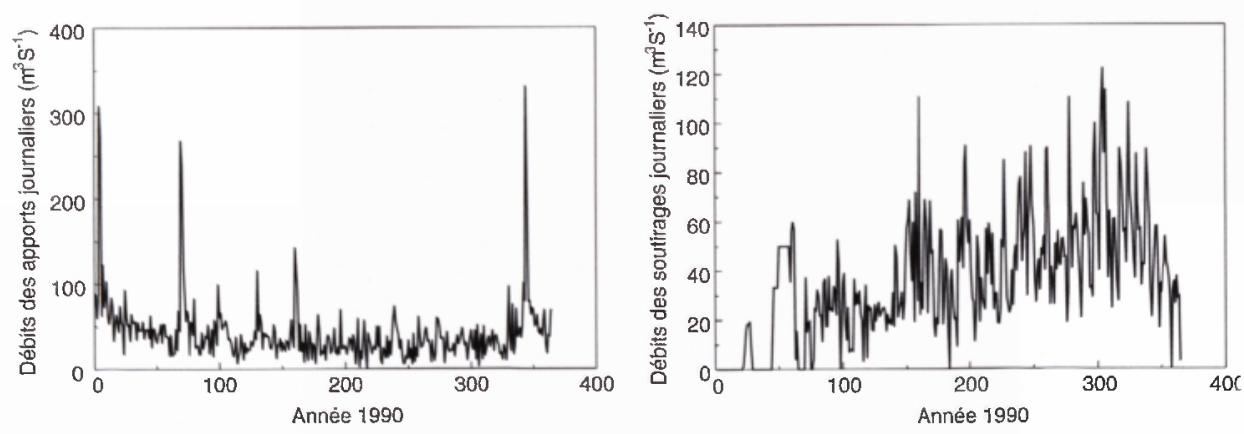

Fig. 4 - Débits journaliers des apports et soutirages de l'année 1990.

Fig. 4 - Inflow and outflow in 1990. 


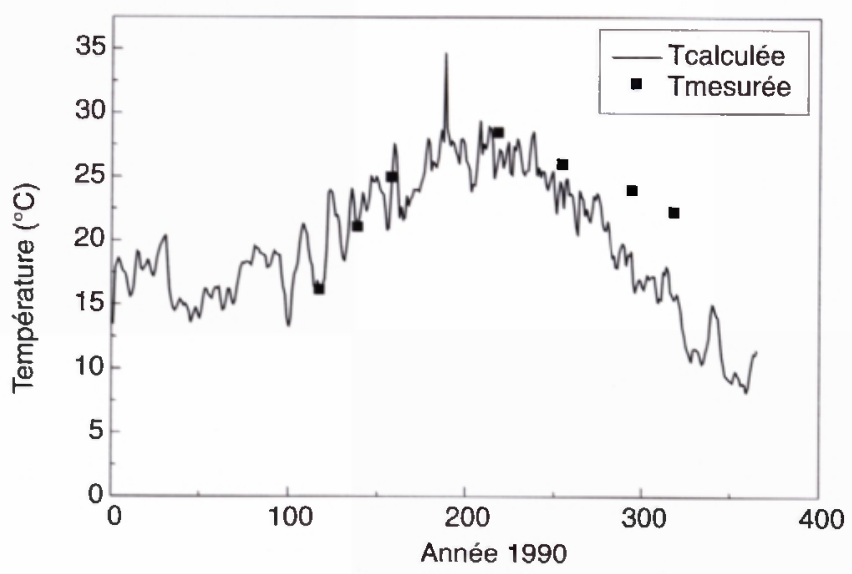

Fig. 5 - Comparaison entre la température des apports calculée par le modèle en rivière et les valeurs mesurées sur le terrain (année 1990).

Fig. 5 - Comparison of calculated and measured affluent temperature.

\section{RÉSULTATS DU MODÈLE THERMIQUE}

Le modèle thermique est ici appliqué à la retenue $\mathrm{Al}$ Massira sur la période Janvier à Décembre 1990.

Lors de la mise en équation du modèle mathématique, nous avons eu à utiliser un certain nombre de fonctions et paramètres. La détermination de ceux ci a été faite en tenant compte de leurs ordres de grandeur connus dans la littérature.

La méthode d'ajustement que nous avons adoptée dans notre démarche de modélisation consiste à faire varier les valeurs des paramètres et à comparer qualitativement les résultats du modèle et les données, de façon à obtenir pour chaque paramètre une valeur unique en procédant à un ajustement convenable des résultats du modèle aux données disponibles.
Par la même occasion une étude de sensibilité des paramètres intervenant dans la mise en équation du modèle a été faite, en vue d'identifier les paramètres ayant un rôle primordial dans le régime thermique de l'écosystème retenue.

Le but d'une telle étude est de déterminer jusqu'à quel point la prise en compte ou non d'un phénomène et la précision de sa formulation (fonction et paramètres) peuvent influencer les résultats de cette modélisation.

\subsection{Sensibilité du modèle aux paramètres de répartition de l'énergie cinétique turbulente}

II s'agit des paramètres $n$ et mv qui sont des coefficients de paramétrisation du mode de production de l'énergie cinétique turbulente (ECT). 
Le refroidissement en surface crée des mouvements convectifs des eaux, la structure verticale de densité devenant instable. L'ECT ainsi créée participe aux mécanismes de creusement de la thermocline.

Le paramètre ' $n$ ' représente la part de l'ECT dissipée sous forme de chaleur suite à un refroidissement en surface, $0 \leq n \leq 1$ et le paramètre ' $m v$ ' représente la part de l'énergie née de l'action du vent en surface, qui servira au mélange.

Dans notre cas on s'est attaché à déterminer le couple ( $\mathrm{n}, \mathrm{mv})$ convenant le mieux à notre modèle.

L'analyse des résultats (fig. 6) montre que pour le couple $(n=0.02$ et $m v=0.2$ ), le mélange entre les deux couches par refroidissement en surface s'effectue de façon prématurée. La colonne d'eau est mélangée sur une plus grande partie de l'année. Alors qu'on note une stratification qui dure plus longtemps dans le cas d'une convection non pénétrante ( $n=0$ et $m v=0.002$ ) de même qu'il persiste un gradient de température de plusieurs degrés entre les deux couches.

\subsection{Sensibilité du modèle aux paramètres d'estimation des échanges air-eau}

\subsubsection{Sensibilité au coefficient $d u$ vent ' $a$ '}

Ce coefficient intervient dans le calcul du flux de chaleur latente par le terme $\mathrm{C}(\mathrm{u})$ :

$$
C_{e}=\rho_{a} L C(u)\left(Q_{e}-Q_{a}\right)
$$

$C_{e}$ : flux d'eau évaporé $W \cdot m^{-2}$

$\rho_{\mathrm{a}}$ : Densité de l'air $=1,293 \mathrm{~kg} \cdot \mathrm{m}^{-2}$

$L$ : chaleur latente d'évaporation en J.kg-1

$\mathrm{C}(\mathrm{u})$ : effet du vent, fonction de transfert empirique adaptée pour compenser la différence in situ entre les conditions du vent de la station météorologique et les conditions du vent locales.

Calcul de $\mathrm{C}(\mathrm{u}): \mathrm{C}(\mathrm{u})=\mathrm{a}(1+\mathrm{u})$

$\mathrm{a}$ : paramètre à caler (sans dimension) : $0,0015<a<0,0025$

$\mathrm{u}$ : vitesse du vent à $2 \mathrm{~m}$ d'altitude $\left(\mathrm{m} \cdot \mathrm{s}^{-1}\right)$

Le coefficient ' $a$ ' conditionne fortement le bilan thermique en surface. Avec un coefficient du vent ' $a$ ' élevé $(a=0.002)$, la masse d'eau a tendance à connaître une évaporation importante (fig. 7). Le gradient thermique vertical s'en trouve réduit, ce qui favorise les échanges entre les deux couches et donc le niveau de la thermocline est situé plus en profondeur. L'inverse est observé lorsqu'on diminue le flux de chaleur latente.

\subsubsection{Sensibilité au coefficient de frottement ' $c d$ '}

L'effet du vent est quantifié par le coefficient de frottement 'cd' qui intervient dans l'estimation de l'énergie cinétique turbulente engendrée par le cisaillement du vent en surface.

Nous avons testé plusieurs valeurs du coefficient 'cd'. On remarque que pour un coefficient important $(\mathrm{cd}=$ 
$-A-$

A: Evolution des températures calculées et mesurées Tsurf : Température calculée en surface.

Tfond : Température calculée au fond.

Msurf : Température mesurée en surface.

Mfond: Température mesurée au fond.
-B-

B : Evolution de la stratification verticale Prof tot: Profondeur totale de la retenue. Prof th: Profondeur de la thermocline.

$\mathrm{n}=0.02, \mathrm{mv}=0.2$
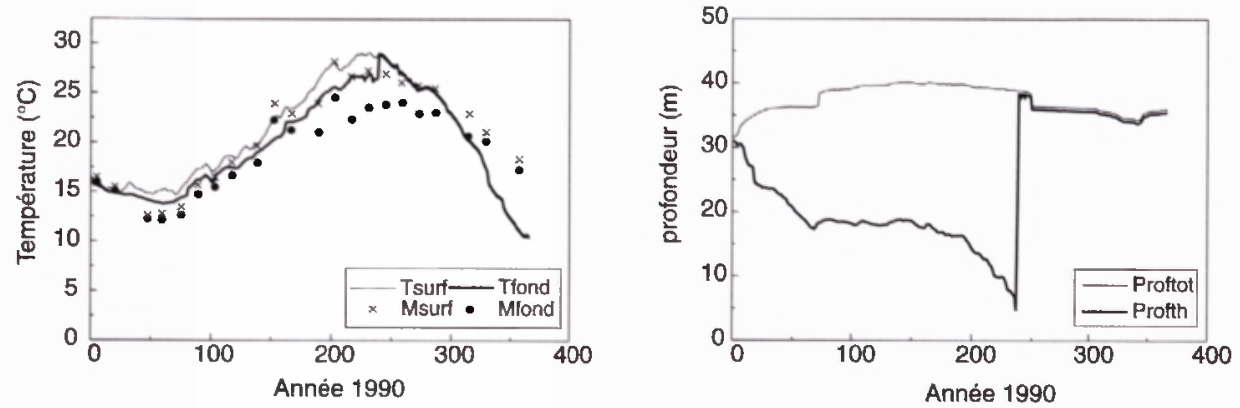

$n=0, m v=0.002$
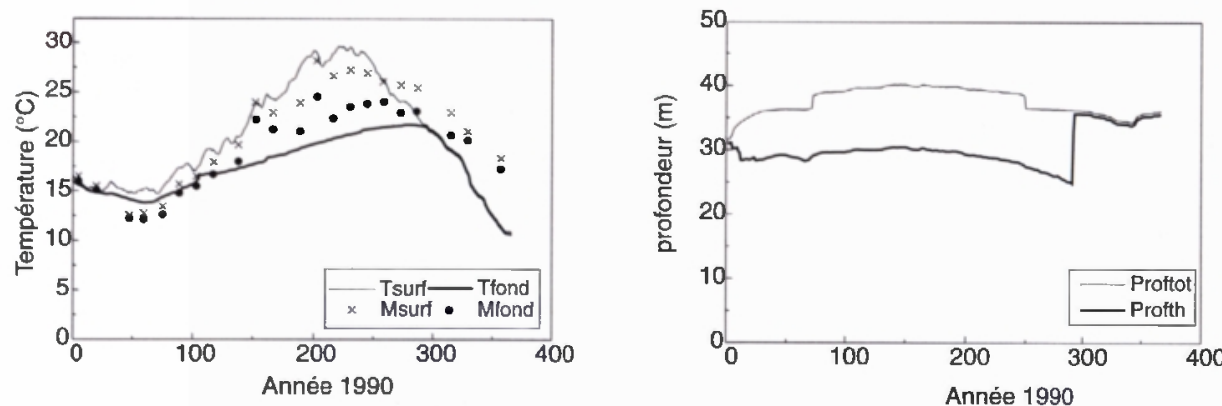

Fig. 6. - Sensibilité du modèle thermique aux coefficients ' $n$ ' et ' $m v$ '.

Fig. 6. - Effect of the ' $n$ ' and 'mv' coefficients on the thermal model.

$2.510^{-2}$ ) (fig. 8), correspondant à un fort effet du vent, :

- la couche de mélange est alors épaisse,

- la thermocline se situe plus en profondeur,
- et le gradient de température entre la surface et le fond s'en trouve diminué.

C'est donc un facteur qui conditionne fortement la stratification, plus particulièrement la position de la thermocline. 
$-A$ -

A : Evolution des températures calculées et mesurées Tsurf : Température calculée en surface. Tfond : Température calculée au fond.

Msurf : Température mesurée en surface.

Mfond : Température mesurée au fond.
-B-

B : Evolution de la stratification verticale Prof tot: Profondeur totale de la retenue. Prof th: Profondeur de la thermocline.

$a=0,002$
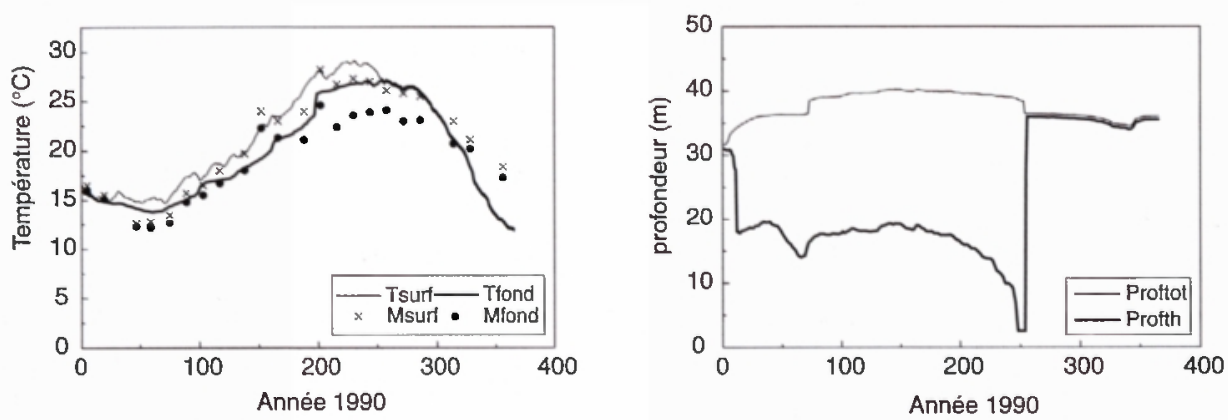

$a=0.0013$
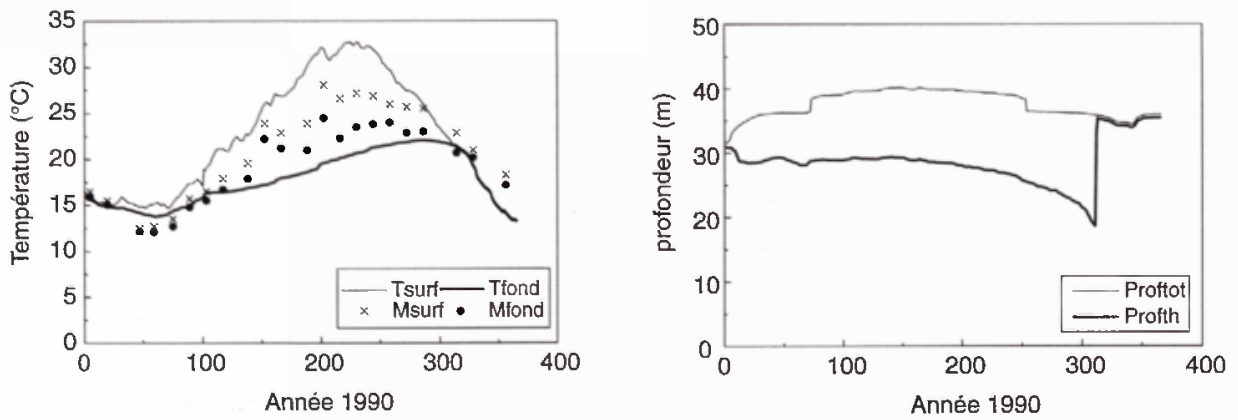

Fig. 7. - Sensibilité du modèle thermique au coefficient du vent 'a'.

Fig. 7. - Effect of the ' $a$ ' wind coefficient on the thermal model.

\subsection{Sensibilité du modèle au coefficient d'extinction de la lumière 'eta'}

L'absorption du rayonnement solaire dans l'eau suit une loi plus ou moins complexe dépendante de la qualité de l'eau. L'expression globale de cette absorption, couramment utilisée est de la forme exponentielle suivante :

$$
R z=R s e^{(e t a * z)}
$$

Où 
-A-

A : Evolution des températures calculées et mesurées Tsurf : Température calculée en surface. Tfond: Température calculée au fond. Msurf : Température mesurée en surface. Mfond : Température mesurée au fond.
$-B-$

B : Evolution de la stratification verticale Prof tot: Profondeur totale de la retenue. Prof th : Profondeur de la thermocline.

$\mathrm{cd}=2.510^{-3}$
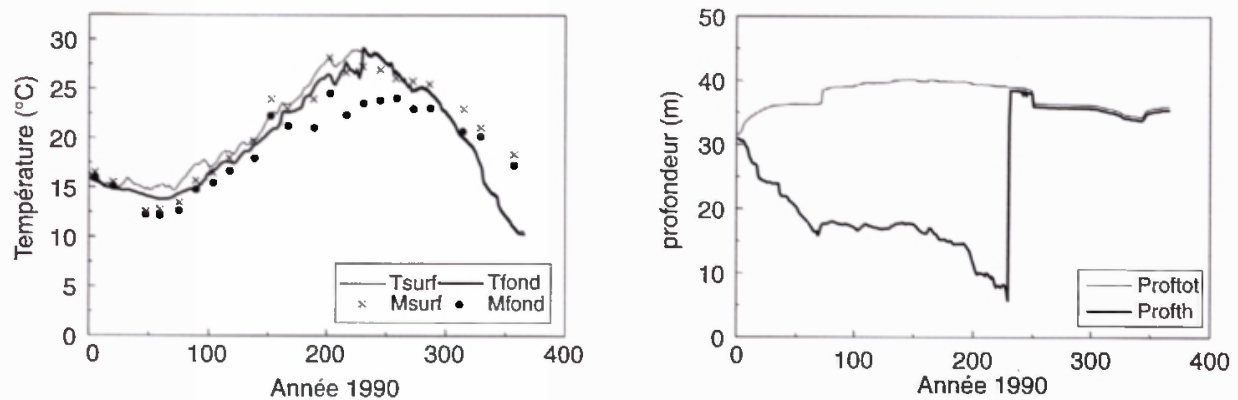

Fig. 8. - Effet d'un coefficient élevé du vent 'cd' sur le modèle thermique.

Fig. 8. - Effect of a high wind coefficient 'cd' on the thermal model.

$\mathrm{Rz}\left(\mathrm{W} \cdot \mathrm{m}^{-2}\right)$ : rayonnement solaire à la profondeur $z$.

Rs $\left(W \cdot \mathrm{m}^{-2}\right)$ : rayonnement solaire à la surface du plan d'eau.

$Z(m)$ : profondeur $Z<0$.

eta $\left(\mathrm{m}^{-1}\right)$ : coefficient d'extinction de la lumière.

Ne disposant pas de mesures du coefficient d'extinction, qui est une variable de forçage, nous avons procédé à une étude de sensibilité. Cette étude (fig. 9) a montré que pour une faible valeur de eta (eta=0.18), la colonne d'eau est complètement mélangée. L'eau étant claire pour les faibles valeurs de eta, le réchauffement de la colonne d'eau du haut vers le bat se fait rapidement et uniformément; les deux couches aboutissent très tôt à des températures égales. Une stratification peu marquée est très rapidement détruite.

Inversement plus la valeur de eta augmente (eta $=1.5$ ), plus la stratification est marquée et dure plus longtemps. On note également un gradient de température plus important entre la couche de surface et la couche de fond.

\subsection{Calage du modèle sur l'année 1990}

Les paramètres qui interviennent dans la formulation des échanges eau-atmosphère et de la vitesse d'en- 
$-A-$

A : Evolution des températures calculées et mesurée Tsurf : température calculée en surface. Tfond: température calculée au fond. Msurt : température mesurée en surface. Miond : température mesurée au fond.

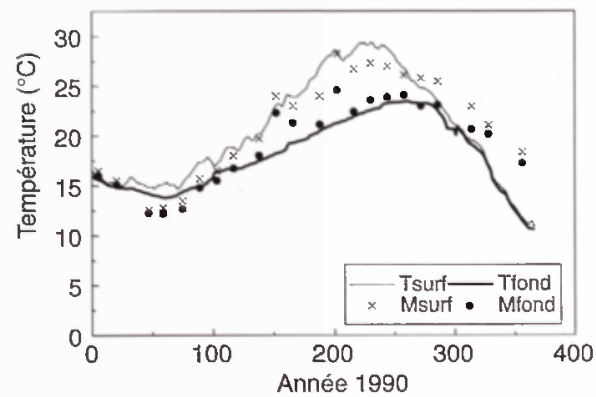

eta $=1.5$

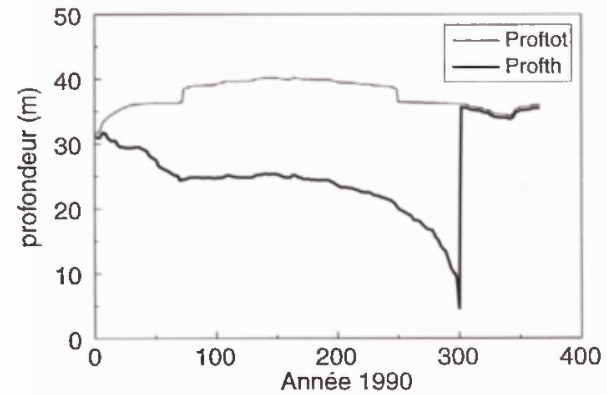

eta $=0.18$
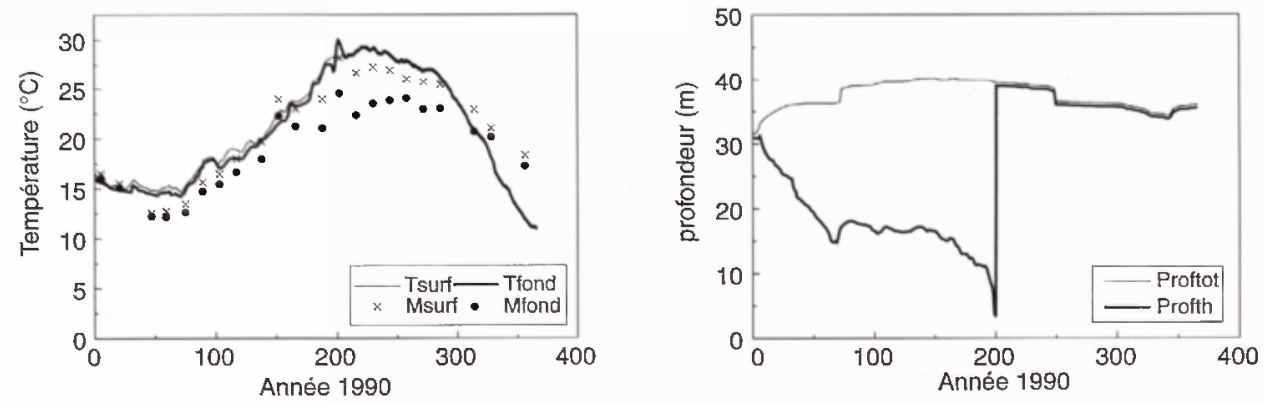

Fig. 9. - Sensibilité du modèle thermique au coefficient d'extinction de la lumière 'eta'.

Fig. 9. - Effect of the light extinction coefficient 'eta' on the thermal model.

traînement sont calés sur la base de données de l'année 1990 (fig.10). Les valeurs retenues pour ces paramètres sont :

$\mathrm{n}=0.014$
$\mathrm{mv}=0.1$
$\mathrm{a}=0.0018$
$\mathrm{~cd}=1.1210^{-2}$
eta $=0.5$

$B$ : Evolution de la stratification verticale Prof tot : profondeur totale de la retenue. Prof th : profondeur de la thermocline. 
$-A$ -

A : Evolution des températures calculées et mesurée Tsurf : température calculée en surface. Tfond : température calculée au fond. Msurf : température mesurée en surface. Mfond : température mesurée au fond.
$-\mathrm{B}-$

$B$ : Evolution de la stratification verticale Prof tot: profondeur totale de la retenue. Prof th : profondeur de la thermocline.
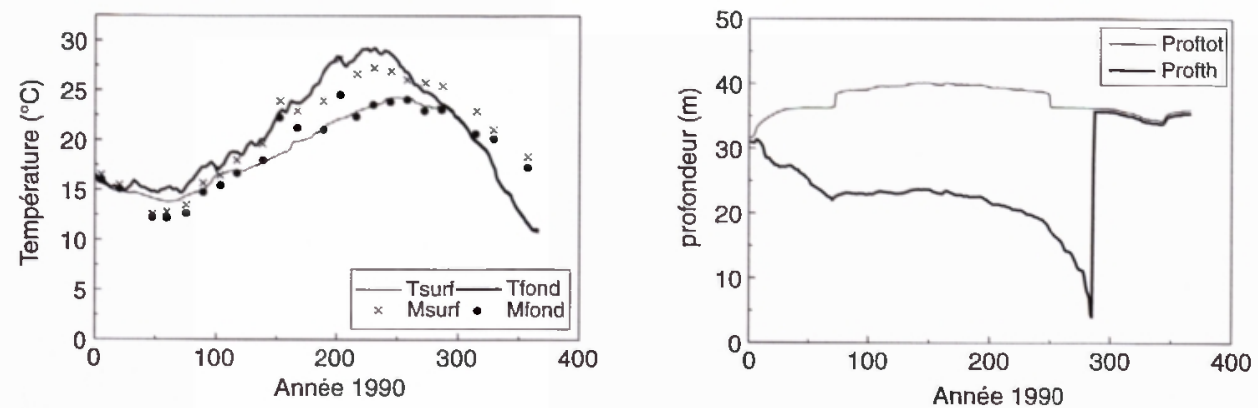

Fig.10. - Calage du modèle sur la base de données de l'année 1990.

Fig.10. - Validation of the model in 1990.

On note qu'au début de l'année, la température est homogène sur toute la colonne d'eau. Au mois de février, la température de surface commence à augmenter alors que celle de la couche profonde augmente légèrement. Au mois de juillet, le gradient thermique entre la surface et le fond atteint son maximum (stratification thermique). Le système s'homogénéise complètement au mois d'octobre.

Les résultats présentés montrent une adéquation satisfaisante entre les températures calculées et celles mesurées dans la retenue Al Massira. La description des températures à la fin de l'année montre des décalages avec les données. Ces écarts des températures observés à la fin de l'année pourraient s'expliquer par le fait que le modèle thermique des ri- vières sous-estime la température des entrées à cette même période.

\section{APPLICATION DU MODÈLE POUR LES ANNÉES 1991 ET 1997}

Dans cette étape, à travers de nouvelles simulations avec le modèle thermique, nous avons testé un autre jeu de données sans modifier la paramétrisation adoptée précédemment. Ainsi, une application du modèle thermique a été réalisée sur les années 1991 et 1997. Cette étude est réalisée en utilisant comme données d'entrées les données météorologiques et les débits respectifs des années 1991 et 1997. Comme pour l'année 1990, la température des apports a été calculée par le modèle thermique des rivières avec les don- 
$-A-$

A : Evolution des températures calculées et mesurée Tsurf : température calculée en surface.

Tfond : température calculée au fond.

Msurf : température mesurée en surface.

Mfond : température mesurée au fond.
$-B-$

$B$ : Evolution de la stratification verticale Prof tot: profondeur totale de la retenue. Prof th : profondeur de la thermocline.
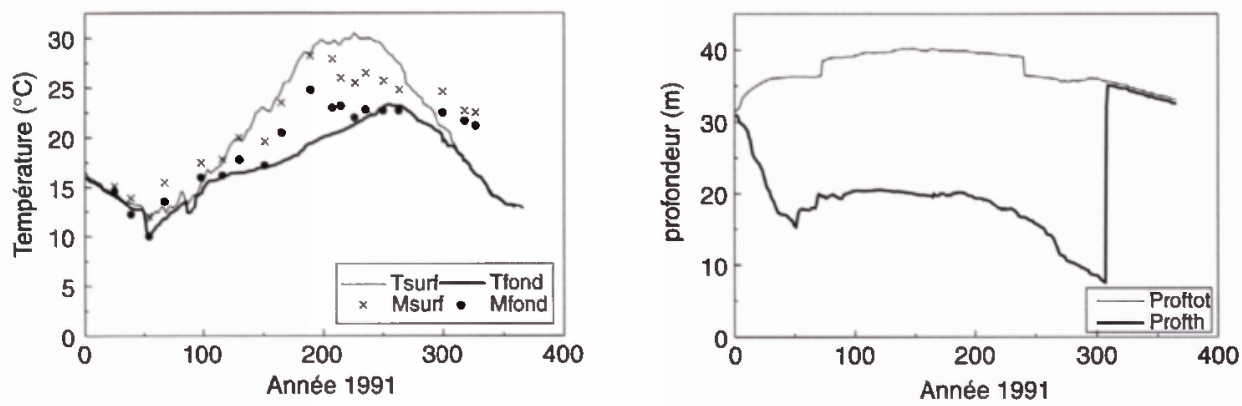

Fig. 11. - Application du modèle pour l'année 1991.

Fig. 11. - Model implementation in 1991.

$-A-$

A : Evolution des températures calculées et mesurée Tsurf : température calculée en surface.

Tfond : température calculée au fond.

Msurt : température mesurée en surface.

Mfond : température mesurée au fond.
-B-

B : Evolution de la stratification verticale Prof tot : profondeur totale de la retenue. Prof th : profondeur de la thermocline.
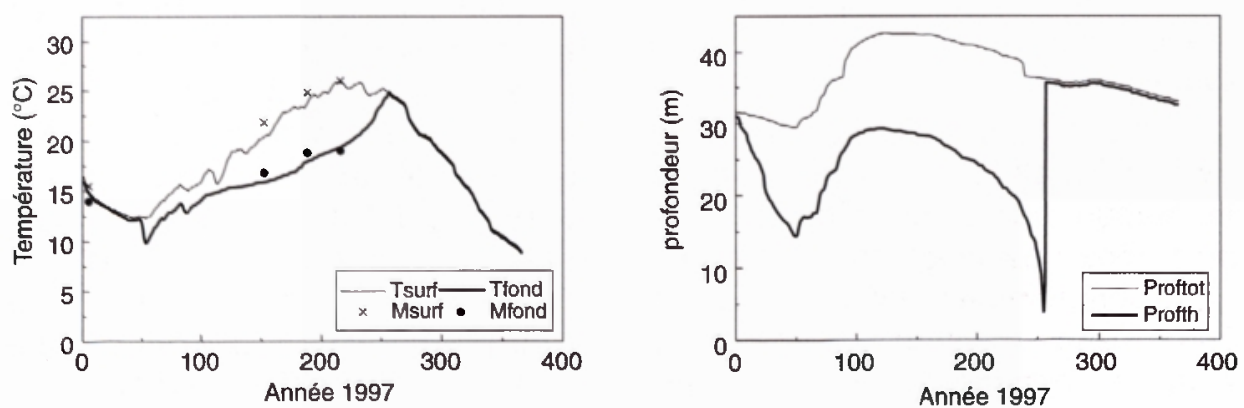

Fig. 12. - Application du modèle à l'année 1997.

Fig. 12. - Model implementation in 1997. 
nées météorologiques respectivement des années 1991 et 1997. Cette phase de validation nécessaire permet, en effet, de rendre compte de l'importance des données initiales d'entrée utilisées dans la qualité des résultats obtenus par le modèle.

Les résultats de l'étape de validation sont présentés sur la figure 11 et 12 , où le modèle élaboré permet de décrire globalement l'évolution de la structure thermique ainsi que les échanges hydrodynamiques au sein de la retenue. On note cependant en automne, une légère différence entre les températures calculées par le modèle et celles mesurées.

\section{CONCLUSION}

Le modèle thermique ainsi présenté s'appuie sur l'ensemble des données recueillies sur le site et sur les variables de forçage (géométrie, météorologie, débits d'entrées et sorties et température des apports). L'hydrodynamique est décrite par un modèle bicouche à bilan d'énergie. Le modèle ainsi développé, calé avec les données de 1990, a permis de reconstituer l'évolution saisonnière de la stratification thermique pour cette année. L'étape de validation pour les années 1991 et 1997 dont les conditions climatologiques et hydrologiques sont différentes montre que le modèle reproduit globalement l'évolution interannuelle du régime thermique dans la retenue Al Massira.
Nous avons donc un outil qui ne peut prétendre être précis étant donné que la structure thermique verticale est naturellement non bicouche. Cependant il a l'avantage d'être simple et de permettre, tenant compte, des conditions climatiques données, de morphologie donnée et d'un mode de gestion des eaux donné d'une retenue, d'avoir une première appréciation rapide du processus de stratification thermique saisonnière pouvant avoir lieu dans cet écosystème.

Il est évident qu'au niveau physique des masses d'eau, beaucoup d'approximations sont faites mais le rôle primordial joué par les échanges eau-atmosphère dans le régime thermique de cet écosystème et le long temps de séjour des eaux dans la retenue nous garantit un résultat acceptable.

La prochaine étape concernera le couplage avec un modèle biologique dont les principales variables sont le phosphore, le phytoplancton et le zooplancton.

\section{REMERCIEMENTS}

Nous adressons nos vifs remerciements à MM. M.Malki et A.Fahd, Professeurs à la Faculté des Sciences Aïn Chock de Casablanca, pour nous avoir fourni des données importantes dans le présent travail. 


\section{RÉFÉRENCES BIBLIOGRAPHIQUES}

Ababou, B., 1995. Elaboration d'un modèle bicouche d'eutrophisation de lacs et réservoirs. Thèse de 3ème cycle. Faculté des Sciences Chouaib Doukkali El Jadida.

Abouzaid, M.H., Foutlane, A. ef Bourchich, L., 1987. Qualité de l'eau de la retenue Al Massira. Naturaliste can (Rev. Ecol. Syst).

Agoumi, A., 1982. Modélisation du régime thermique de la Manche. Thèse de Doctorat; Ecole Nationale des Ponts et Chaussées.

Agoumi, A., 1985. Modélisation de l'écosystème pélagique en manche. Etude de l'influence des phénomènes physjques sur le système planctonique. Thèse Doct. Etat. ENPC. Paris.

Agoumi, A., 1991. Modèle d'eutrophisation de la retenue Al massira. Congré Mondiale de l'Eau. Rabat 1991.

Bensaid, F.Z., 1994. Contribution à la modélisation mathématique de la qualité des eaux de l'oued Oum Er Rabia. Thèse de 3ème cycle. Faculté des Sciences Semlalia Marrakech.

Bensaid, F.Z., Agoumi A. et Aboufirassi M., 1994. Impact des unités sucrières sur la qualité des eaux de l'oued Oum $\mathrm{Er}$ Rbia. Acte de la $4^{\text {ème }}$ Conférence Internationale de Limnologues d'Expression Française. Marrakech du 25 au 28 avril, 1994.

Cazelle, B., 1987. Modélisation d'un écosystème lotique: Dynamique du carbone organique dissous et des microorganismes bentiques dans un cours d'eau pollué. Thèse de Doctorat. Université Claude Bernard-Lyon I.

Dafir, J.E., 1997. Application de la dynamique du phosphore à l'étude de l'organisation et du fonctionnement des écosystèmes aquatiques. Thèse Doct. Etat Es-Sciences Géochimie et Environnement. Faculté des Sciences Ain Chok Casablanca.

Delclaux, F., 1980. Production primaire en milieu thermiquement stratifié. Modélisation et application à la rivière Lot. Thèse de Doctorat. Institut National Polytechnique de Toulouse.

Direction de la Recherche et de la Planification de l'Eau., 1990. Programme de surveillance de la qualité des eaux. Rabat.

Emberger, J., 1955. Une classification biogéographie des climats. Rev. Trav. Botan et Zool. Faculté des sciences. Montpellier. Ser. Botan.

Fahde, A., 1994. Etude des peuplements de Cladocères et Rotifères dans le lac de barrage Al Massira (Maroc). (Hétérogénéité horizontale, dynamique des populations, broutage). Thèse Doct. Etat Es-Sciences Biologie, option: Ecologie- Hydrobiologie.

Lesaos, J.P., 1979. Simulations numériques de l'évolution de la couche superficielle de l'océan et confrontation avec des mesures 'in situ'. Thèse de $3^{\text {ème }}$ cycle, océanographie-physique. Université de Bretagne occidentale.

Malki, M., 1994. Etude de la communauté phytoplanctonique et des caractéristiques physico-chimiques des eaux du lac-réservoir Al massira. Thèse de Doct. Etat Es-Sciences. Biologie. Faculté des Sciences Aïn Chock. Casablanca.

Moncef, M., 1993. Etude du peuplement de Copépodes et des larves de Chaoboridae de la retenue de barrage $\mathrm{Al}$ Massira (Maroc): Dynamique des populations, Répartition spatio-temporelle, Temps de développement, Production secondaire, Migrations nycthémérales, Broutage. Thèse. Doct. Etat Es-Sciences Naturelles, option: Ecologie. Faculté des Sciences Aïn Chock. Casablanca. 
Moncef, M., Malki, M., Fahde, A. et Devaux J., 1994. Mesure de l'intensité et de la sélectivité du broutage zooplanctonique diurne et nocturne sur le peuplement phytoplanctonique de la retenue Al Massira (Maroc). Revue des Sciences de l'Eau.

Salençon, M.J., 1986. Modélisation de l'eutrophisation des lacs et retenues. EDF,Rapport HE 31/86.08.
Salençon, M.J., 1995. Stratification thermique d'un réservoir : Modèle à bilan d'énergie, EOLE, Rapport HE. 31/94/001,Electricité de France.

Thébault, J.M. and Salençon, M.J., 1993. Simulation model of a mesotrophic reservoir (Lac de Pareloup) : biological model. Ecol. Modelling, 65 :1-30. 